Polymer Journal, Vol. 9, No. 5, pp 489-497 (1977)

\title{
A Kinetic Study of the Cooperative Binding of Toluidine Blue to Poly(acrylic acid)
}

\author{
Hidetoshi Ushio, Hitoshi Nishimura, Yoshikuni Tsuji, \\ Takayuki SANO, and Tatsuya YAsUNAGA \\ Department of Chemistry, Faculty of Science, \\ Hiroshima University, Hiroshima 730, Japan.
}

(Received March 22, 1977)

\begin{abstract}
Kinetic studies have been carried out on the cooperative binding of toluidine blue to poly(acrylic acid) by the electric-field pulse method in order to compare the binding property with that for poly $(\alpha-\mathrm{L}$-glutamic acid). Based on the results of our experiments in the coupling of the aggregation process of the dye to the nucleation process, we were able to arrive at a mechanism which we introduce in this paper. The association rate constant of the dye aggregation process was found to be about one order of magnitude smaller than that for the poly $(\alpha-\mathrm{L}-\mathrm{glutamic}$ acid $)$. The reason for this is the difference in the polymer structures following hydration. Also the effect of the degree of ionization of a polymer on the various properties of the binding of a dye was also examined. It was found consequently that the bound dyes became more stable and compact with an increase in the degree of ionization.

KEY WORDS Kinetics / Cooperative Binding / Poly(acrylic acid) / Nucleation Process / Aggregation Process / Electric-Field Pulse Method /
\end{abstract}

A number of studies have been carried out to elucidate the equilibrium properties of the interaction of the basic dye with the polymer. ${ }^{1-5}$ This system is of interest as an available model of the interaction of small molecules with biopolymers in vivo.

Many kinetic studies on the macromolecular binding have also been performed with the synthetic polymer and nucleic acid, etc. ${ }^{6-9}$ In early works, the reaction mechanism of the dye binding to the polymer has been interpreted by one similar to the reaction which occurs between the small molecules. However, by equilibrium experiments, it was also known that the dye-dye interaction occurs on the surface of the polymer. ${ }^{2}$ Therefore the theory of a cooperative binding taking into account the nearest-neighbor interaction of ligands has been presented ${ }^{10,11}$ and has been applied successfully to certain macromolecular systems. ${ }^{12-15}$

The binding mode of the basic dyes to anionic polymers has been generally considered to be of an essential electrostatic nature and the binding process has been attributed to the diffusion-controlled process for the $\operatorname{poly}(\alpha$-Lglutamic acid) $\left[(\mathrm{Glu})_{n}\right]^{13,16}$ and poly(acrylic acid) $(\mathrm{PAA})^{14}$ (about $10^{9} \mathrm{M}^{-1} \mathrm{sec}^{-1}$ ). For the other polymers (e.g., $\left.\mathrm{DNA}^{8}\right)$, however, a somewhat slow process has been found in the association of the dye to the polymer (about $10^{7} \mathrm{M}^{-1} \mathrm{sec}^{-1}$ ). Furthermore, the authors previously discovered the difference in the binding properties of the helical and the coiled forms of $(\mathrm{Glu})_{n} \cdot{ }^{16}$ Thus if the binding behaviors of these association processes reflect the specificity of the polymer, the cause for the difference in these processes among the polymers should warrant investigation.

In the work reported here, the interaction of the basic dye of toluidine blue with PAA which has been chosen as the fundamental macromolecule having no helix-coil transition, is studied, using the electric-field pulse method. The kinetic studies of the interaction between polymer and dye have been carried out mainly by the temperature-jump method. This method requires a high salt concentration not suitable 
to this kind of system since it prevents for the most part binding of the dye to the polymer. The electric-field pulse method, based on the dissociation field effect, requires no salt and is of considerable advantage to the study of an ionic interaction such as the polymer-dye system.

The result was compared with that for the toluidine blue-(Glu $)_{n}$ system, ${ }^{16}$ and the dependency of kinetic and equilibrium properties of the present system on the degree of ionization was also examined in order to obtain more detailed information on the electrostatic nature of the binding.

\section{EXPERIMENTALS}

\section{Materials}

Toluidine blue was purified in the manner described previously. ${ }^{16}$ Fresh stock solutions of toluidine blue were prepared for each experiment.

Poly(sodium acrylate) (NaPAA) was synthesized from acrylic acid monomer in order to obtain much the same degree of polymerization $(D P)$ as that of $(\mathrm{Glu})_{n}$ in the previous work. ${ }^{16}$ With distilled water and ammonium persulfate as the initiator, an aqueous monomeric acrylic acid was put into the hard glass tube with sufficient nitrogen and then polymerized at $50 \pm 0.1^{\circ} \mathrm{C}$ for $72 \mathrm{hr}$. The initial value of the $\mathrm{pH}$ was 4.5 which was chosen so as to obtain a suitable degree of polymerization. After this PAA was dialyzed against the deionized water for five days, the sodium form of PAA was obtained by reprecipitating three times with sodium hydroxide and ethanol. Furthermore the NaPAA was dialyzed sufficiently and dried in vacuum for $12 \mathrm{hr}$ at $60^{\circ} \mathrm{C}$ after being lyophilized. The value of the $\mathrm{pH}$ of the aqueous NaPAA solutions was 8.84 , confirming that the sodium form of PAA had been obtained. Elementary analysis, infrared spectrum and nuclear magnetic resonance spectrum were used for identification. The elementary analysis showed that one molecule was contained per two residues of NaPAA. The intrinsic viscosity $[\eta]$ was determined in order to evaluate the value of the $D P$, using Kagawa's relation ${ }^{17}$

$$
[\eta]=6.52 \times 10^{3}(D P)^{0.64}
$$

and it was found to be about 940. All other chemicals used in this experiment were of reagent grade.

Since the ionic strength of a sample solution has to be low enough to yield the exact rectangular pulse, a buffer was not used in the kinetic experiments. Also, in spectrophotometric experiments, a buffer was not used to maintain the same condition as that for the kinetic experiments. Sample solutions were prepared by adding aqueous toluidine blue solution to an aqueous PAA solution with stirring; the value of $\mathrm{pH}$ was then adjusted to the desired value by addition of hydrochloric acid or aqueous sodium hydroxide. All measurements were performed at three $\mathrm{pH}$ regions of $6.5,8$, and 10 in order to examine the dependences of the binding properties upon the degree of ionization of PAA.

\section{Method}

Kinetic measurements were carried out using the electric-field pulse technique in which the instruments were virtually the same as described elsewhere. ${ }^{16,18}$ A wavelength of $635 \mathrm{~nm}$ corresponding to the absorption maximum of toluidine blue ${ }^{19}$ was employed to monitor the reaction progress. It is known that the application of an electric field to the macromolecule causes orientation of the macromolecule toward the direction of the field. ${ }^{20}$ Thus, to cancel out the contribution of this orientation effect on the chemical relaxation signal, a polarizer was inserted between the cell and monochrometer. The angle determined for the polarizer that would minimize the signal amplitude at the isosbestic wavelength was about 75 degrees to the direction of the electric field.

The Union Giken SM-401 spectrophotometer was used for the spectrophotometric measurements. The $\mathrm{pH}$ was measured with HitachiHoriba type F-5 pH meter with an accuracy of $\pm 0.2 \mathrm{pH}$ unit. The procedure for the $\mathrm{pH}$ titration has been described in our previous work. ${ }^{16}$ In the kinetic experiments, the temperature was controlled at $20^{\circ} \mathrm{C}$ by circulating water from an external thermostat. All other measurements were made at room temperature with the exception of the titration experiments, being at $20^{\circ} \mathrm{C}$. 


\section{RESULTS AND DISCUSSION}

In order to determine the degree of ionization of PAA at various $\mathrm{pH}, \mathrm{pH}$ titration was performed for the PAA and PAA-toluidine blue complex. The total concentration of the titrant added was the same with the initial concentration of PAA in the cases of both the presence and absence of toluidine blue. This indicates that all carboxylic groups in PAA are titrated and the masking effect of carboxylic group by toluidine blue does not occur. This fact also explains the experimental result of the titration curve for PAA-toluidine blue complex being identical with that for PAA. The ionization degree values were $0.4,0.8$, and 1.0 at $\mathrm{pH}$ $6.5,8,10$, respectively, which are in fairly good agreement with the values in the literature. ${ }^{21}$

Various equilibrium parameters were determined by the procedures used by Schwarz. ${ }^{10,12}$ Since the dye used in this work is the same as those in our previous work, ${ }^{16}$ the equilibrium constant for dimerization of toluidine blue are shown only in Table I.

The molar extinction coefficient of stacked dye to the polymer $\left(\varepsilon_{\mathrm{st}}\right)$ is obtained using eq 2

$$
\varepsilon=\varepsilon_{\mathrm{st}}+\left(\varepsilon_{\mathrm{A}}-\varepsilon_{\mathrm{st}}\right) \frac{s}{K}\left(c_{\mathrm{A}}{ }^{0}\right)^{-1}
$$

where $\varepsilon$ is the apparent extinction coefficient of the sample solutions, $\varepsilon_{\mathrm{A}}$ is the extinction coefficient of the free monomeric dye, $K$ is the cooperative binding constant, $c_{\mathrm{A}}{ }^{0}$ is the total weighing-in concentration of dye and $s=K \bar{c}_{\mathrm{A}}$ $\left(\bar{c}_{\mathrm{A}}=\right.$ the equilibrium concentration of the free monomeric dye). If the parameter $s$ becomes almost constant with changes in $c_{\mathrm{A}}{ }^{0}$, a plot of $\varepsilon v s .\left(c_{\mathrm{A}}{ }^{0}\right)^{-1}$ at constant polymer-to-dye ratio $(P / D)$ should eventually give a straight line, and the value of $\varepsilon_{\mathrm{st}}$ should be determined from the intercept on the ordinate axis. These plots are shown in Figure 1.

Next, the number of binding sites per residue $(g)$ of PAA and the cooperative binding constants $(K)$ were determined using the following relations

$$
\begin{gathered}
\gamma_{\mathrm{A}}{ }^{*}+\theta g P=1 \\
K=\left(\dot{\gamma}_{\mathrm{A}}^{*}{ }^{*} c_{\mathrm{A}}^{0}\right)^{-1}+2 K_{\mathrm{d}}
\end{gathered}
$$

where $\gamma_{A}{ }^{*}$ is the fraction of free dye, $\theta$ is the frac-

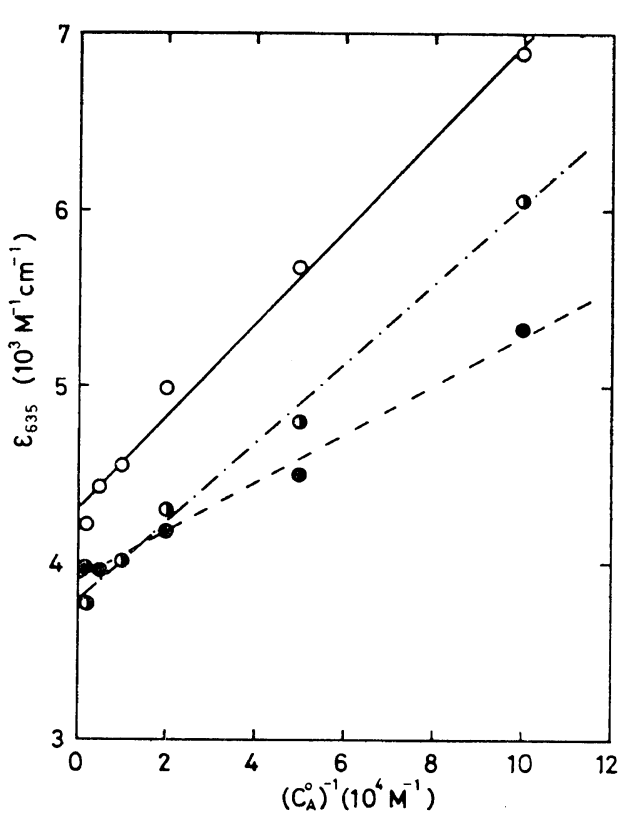

Figure 1. Plots of the apparent molar extinction coefficient, $\varepsilon_{635}, v s$. the reciprocal value of the total weighing-in concentration of the dye, $\left(1 / c_{\mathrm{A}}{ }^{0}\right)$, at constant polymer-to-dye ratio $P / D=5 ; \bigcirc \mathrm{pH} 6.5$; (), $\mathrm{pH} 8.0 ; 0, \mathrm{pH} 10$.

tion of occupied binding sites, $K_{\mathrm{d}}$ is the dimerization constant of dye and $P \equiv P / D$. Plotting $\gamma_{\mathrm{A}}{ }^{*} \nu s$. $P / D$ gives a binding curve shown in Figure 2, satisfying eq 3 . The common-limiting straight line for small $P$ values may be extrapolated to the $P$ axis where the intercept corresponds to $g P=1$ or $P=g^{-1}$. From the value of $\gamma_{A}{ }^{*}$ at the intersection point $\left(\dot{\gamma}_{\mathrm{A}}^{*}\right)$ between the binding curve and the second auxiliary straight line having half the slope of the first one, the value of $K$ can be determined using eq 4 . Thus at the $\mathrm{pH}$ $6.5,8$, and 10 , the values of $g$ were $1.1,1.2$, and 1.4, and the values of $K$ to be $4.8 \times 10^{5} \mathrm{M}^{-1}$, $5.4 \times 10^{5} \mathrm{M}^{-1}$, and $1.7 \times 10^{6} \mathrm{M}^{-1}$, respectively. The value of $g$ was determined assuming that all residues of PAA could be supplied as the binding site. However, at the $\mathrm{pH}$ of 6.5 and 8 , the carboxylic groups are partially ionized and the residues of PAA exist in two forms of ionized and un-ionized carboxylic group. Therefore, in contrast with the above assumption, assuming that the toluidine-blue molecules bind only to ionized carboxylic groups, an ionized 
H. Ushio, H. Nishimura, Y. Tsuji, T. Sano, and T. Yasunaga
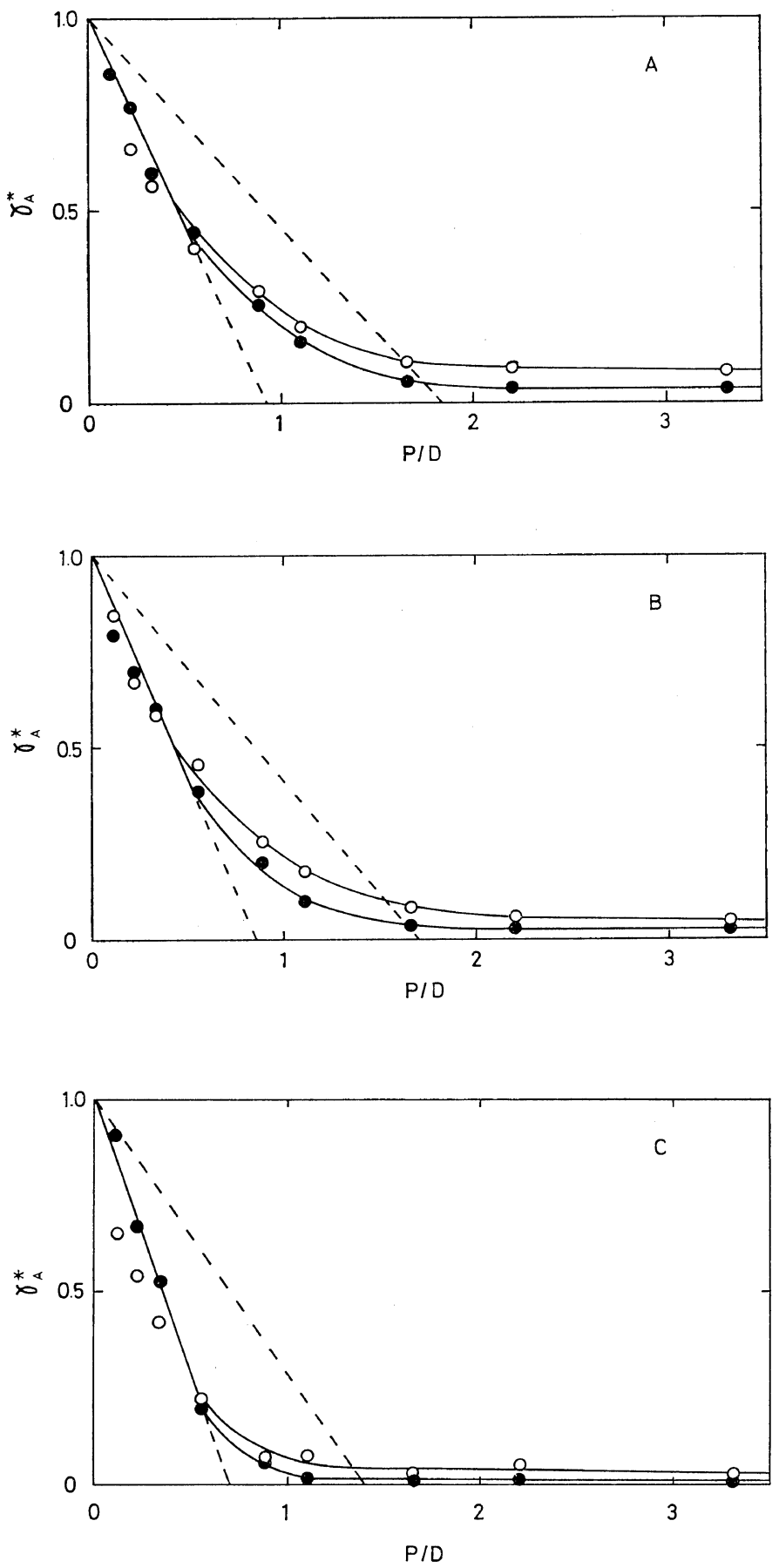

Figure 2. Plots of the fraction of free dye, $\gamma_{A}^{*}$, vs. the polymer-to-dye ratio, $P / D$, at constant total weighing-in concentration of the dye, $c_{\mathrm{A}}{ }^{0}: \bigcirc, c_{\mathrm{A}}^{0}=20 \mu M ; \bigcirc, c_{\mathrm{A}}^{0}=50 \mu M$; (A), $\mathrm{pH}$ 6.5; (B), $\mathrm{pH}$ 8.0; (C), $\mathrm{pH} 10$. The broken straight lines are used to determine the parameters $g$ and $K$ (details are in the text). 
carboxylic group-to-dye ratio should be substituted for the polymer-to-dye ratio of the absissa axis in Figure 2. On this assumption, the values of $g$ turn out to be $2.7,1.5$, and 1.4 at $\mathrm{pH} 6.5,8$, and 10 , respectively. These values indicate that the number of binding sites for the ionized carboxylic groups differs twice between $\mathrm{pH} 6.5$ and 10, which seems to be unlikely in actual practice, taking into account the electrostatic interaction. (The possibility of the dimer of the dye binding to the polymer can be denied because of the negligible concentration of the dimer.) Consequently it seems reasonable in the determination of the value of $g$ to assume that all residues of PAA contribute equally to the binding site.

If the toluidine-blue molecule binds directly to the residue of PAA electrostatically it is expected that some difference in the value of $g$ exists between two binding modes for ionized and un-ionized carboxylic groups. Actually, however, no apparent difference in the value of $g$ was observed between $\mathrm{pH} 6.5$ and 10. As a most plausible binding mode, it may be supposed that the dye binds to the PAA like an ionic atmosphere.

The cooperative parameter $(q)$ was obtained using the following relation.

$$
q \frac{(1-\theta)}{(1-2 \theta)^{2}}=\frac{s}{(1-s)^{2}}
$$

Since this equation does not hold at $\theta=\frac{1}{2}$, the value of $q$ has to be obtained in the region of $\theta<\frac{1}{2}$ (sufficiently large $P / D$ ). The values of $q$ obtained from rough estimations are given in Table I. Generally it is proposed that the cooperativity comes about by the dye-dye interaction on the polymer, so that the value of $q$ may depend on the degree of the overlap of the bound dyes. This dye-dye interaction easily occurs when the charge of the dye is fully neutralized because of the decrease of the electric repulsion force between the dyes. Accordingly it can be understood that $q$ increases with the value of $\alpha$ of PAA, as shown in Table I.

All parameters obtained above are listed in Table I. On the basis of these parameters, the binding scheme of toluidine blue to PAA can be considered as follows: toluidine-blue molecules bind to PAA like an ionic atmosphere, through a Coulombic interaction. With increasing $\alpha$, the charge density around the polymer chain increases and toluidine-blue molecules come to bind more closely to PAA. As a result, the degree of interaction of the dye with the nearest neighbors is also enhanced and the cooperativity is increased. From the comparison of the equilibrium parameters between PAA obtained in the present work and $(\mathrm{Glu})_{n}$-toluidine blue systems, ${ }^{16}$ it is conclusively suggested that the binding properties are greatly affected by the difference in the conformation rather than that in the degree of ionization of the polymer.

A typical kinetic trace in PAA-toluidine blue complex is shown in Figure 3 in which the upward change of the signal corresponds to the increase of toluidine-blue monomer. This figure shows that the toluidine-blue monomer increases during the application of the electric field and decreases with the absence of the field. With the electric-field pulse method by which detection is noted by absorbance change, generally, two relaxation signals having opposite in direction to each other, and which are characterized by the same relaxation time, can be observed in the case when the reaction reaches equilibrium within the pulse duration. ${ }^{22}$ On the other hand, in the case of the reaction not reaching equilibrium within the pulse duration, the analyzable relaxation signal can be observed

Table I. Equilibrium properties of cooperative binding of toluidine blue to poly(acrylic acid) at $20^{\circ} \mathrm{C}$

\begin{tabular}{ccccccc}
\hline $\mathrm{pH}$ & $\begin{array}{c}K_{\mathrm{d}}, \\
10^{3} M^{-1}\end{array}$ & $\begin{array}{c}\varepsilon_{\mathrm{st}}, \\
10^{3} M^{-1} \mathrm{~cm}^{-1}\end{array}$ & \multicolumn{1}{c}{$g$} & $\begin{array}{c}K, \\
10^{5} M^{-1}\end{array}$ & $q$ & $\alpha$ \\
\hline 6.5 & $6.0( \pm 0.7)$ & $4.3( \pm 0.11)$ & $1.1( \pm 0.06)$ & $4.8( \pm 0.2)$ & 20 & 0.4 \\
8 & $6.7( \pm 0.4)$ & $3.8( \pm 0.08)$ & $1.2( \pm 0.1)$ & $5.4( \pm 0.4)$ & 30 & 0.8 \\
10 & $12 \quad( \pm 0.8)$ & $3.9( \pm 0.09)$ & $1.4( \pm 0.06)$ & $17( \pm 4)$ & 80 & 1.0 \\
\hline
\end{tabular}




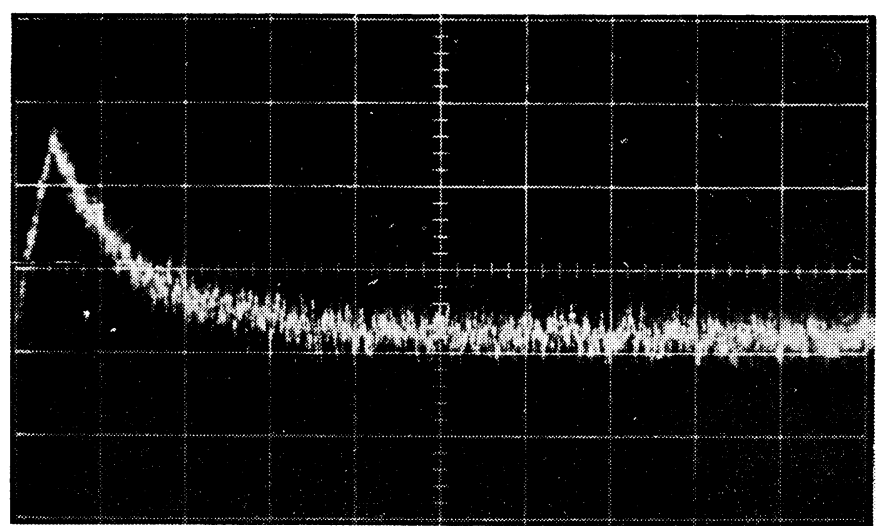

Figure 3. A typical kinetic trace for the interaction of toluidine blue $(50 \mu M)$ with poly(acrylic acid) $(150 \mu M)$ at $\mathrm{pH} 8.0$. The vertical scale in 0.2 volt/division, and the horizontal scale is $50 \mu \mathrm{sec} /$ division. The wavelength used for observation was $635 \mathrm{~nm}$.

only when the electric field is no longer present. In this experiment, as can be seen in Figure 3, no reaction has reached equilibrium within the pulse duration $(20 \mu \mathrm{sec})$ and so only the relaxation spectrum brought on by the removal of the field was used for analysis.

At $545 \mathrm{~nm}$, which corresponds to the absorption of the complex, the relaxation signal having an opposite direction to that obtained at $635 \mathrm{~nm}$ was observed. This fact implies that the observed relaxation phenomena may be due to the binding process of the dye to the polymer. The following three basic reaction mechanisms have been proposed for the cooperative interaction of dye with polymer

$$
\begin{aligned}
& \mathrm{auu}+\mathrm{A} \underset{k_{\mathrm{D}}}{\stackrel{k_{\mathrm{R}}}{\rightleftharpoons}} \mathrm{aau} \\
& \mathrm{uuu}+\mathrm{A} \underset{f_{\mathrm{u} q k_{\mathrm{D}}}}{\stackrel{f_{\mathrm{u}} k_{\mathrm{R}}}{\rightleftarrows}} \mathrm{uau} \\
& \mathrm{aua}+\mathrm{A} \frac{f_{\mathrm{a}} k_{\mathrm{R}}}{\underset{f_{\mathrm{a}}(1 / q) k_{\mathrm{D}}}{\longrightarrow}} \text { aaa }
\end{aligned}
$$

where $k_{\mathrm{R}}$ and $k_{\mathrm{D}}$ are the rate constant for recombination and dissociation process, respectively, and $f_{\mathrm{u}}$ and $f_{\mathrm{a}}$ are defined by Schwarz. ${ }^{10}$ In the case of sufficiently large $q$ the chemical relaxation of binding predominantly reflects the reaction of eq 6 . Thus, the relaxation equation can be written as follows.

$$
\tau^{-1}=2 k_{\mathrm{R}} \frac{g}{\sqrt{q}} \sqrt{ } \theta(1-\theta) P c_{\mathrm{A}}{ }^{0} .
$$

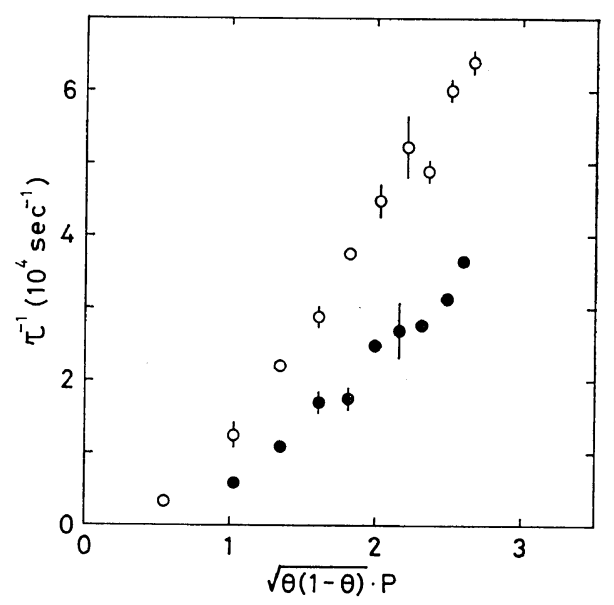

Figure 4. Plots of the reciprocal relaxation time, $(1 / \tau)$, vs. $\sqrt{\theta(1-\theta)} P$ according to the equation (9) at constant total weighing-in concentration of the dye, $c_{\mathrm{A}}{ }^{0}$, at $\mathrm{pH} 10: \bigcirc, c_{\mathrm{A}}{ }^{0}=20 \mu M ; 0, c_{\mathrm{A}}{ }^{0}=50 \mu M$.

According to this equation, Figure 4 shows the plots of the reciprocal relaxation time $\left(\tau^{-1}\right) v s$. $\sqrt{\theta(1-\theta)} P$ at constant $c_{\mathrm{A}}{ }^{0}$. (As a typical example, only the result at $\mathrm{pH} 10$ is shown.) Contrary to our expectations, however, a straight line traversing the origin was not obtained in these plots, indicating that the reaction mechanism for the PAA-toluidine-blue system is somewhat different from the usual one, such as $(\mathrm{Glu})_{n}$-toluidine-blue system. ${ }^{16}$

Another mechanism which takes into account the redistribution process of the bound dye has 
also been proposed for the PAA-proflavine system. ${ }^{14}$ This process is predominant for large values of $P / D$ and was found to be the same mechanism as eq 6 for a sufficiently small $P / D$. Since the present work was performed at a small $P / D$ value the relaxation equation should be the same as eq 9. Accordingly neither can the mechanism for the redistribution process explain the experimental results in the present work.

As a reason for not being able to apply the mechanism of eq 6 , it is felt that the present chemical relaxation of binding might reflect not only the aggregation process of eq 6 but also the nucleation process of eq 7. Accordingly, on the basis of this supposition, the kinetic equation may be written as

$$
\begin{aligned}
-\frac{\mathrm{d} c_{\mathrm{A}}}{\mathrm{d} t}= & 2 k_{\mathrm{R}} c_{\mathrm{auu}} c_{\mathrm{A}}-2 k_{\mathrm{D}} c_{\mathrm{aau}} \\
& +f_{\mathrm{u}} k_{\mathrm{R}} c_{\mathrm{uuu}} c_{\mathrm{A}}-f_{\mathrm{u}} q k_{\mathrm{D}} c_{\mathrm{uau}}
\end{aligned}
$$

where $c$ denotes the concentration of each species. With $x_{\mathrm{A}}=c_{\mathrm{A}}-\bar{c}_{\mathrm{A}}, x_{\mathrm{auu}}=c_{\mathrm{auu}}-\bar{c}_{\mathrm{auu}}$, etc. representing deviations from the new equilibrium concentrations $(\bar{c})$, eq 10 can be transformed to the relaxation equation

where

$$
\begin{aligned}
-\frac{\mathrm{d} x_{\mathrm{A}}}{\mathrm{d} t}= & 2 k_{\mathrm{R}} \bar{c}_{\mathrm{auu}} x_{\mathrm{A}}\left\{1+X_{1}(t)\right\} \\
& +f_{\mathrm{u}} k_{\mathrm{R}} \bar{c}_{\mathrm{uuu}} x_{\mathrm{A}}\left\{1+X_{2}(t)\right\}
\end{aligned}
$$

$$
\begin{aligned}
& X_{1}(t)=\left(\frac{x_{\text {auu }}}{\bar{c}_{\text {auu }}}-\frac{x_{\text {aau }}}{\bar{c}_{\text {aau }}}\right) \frac{\bar{c}_{\mathrm{A}}}{x_{\mathrm{A}}} \\
& X_{2}(t)=\left(\frac{x_{\text {uuu }}}{\bar{c}_{\text {uuu }}}-\frac{x_{\text {uau }}}{\bar{c}_{\text {uau }}}\right) \frac{\bar{c}_{\mathrm{A}}}{x_{\mathrm{A}}}
\end{aligned}
$$

At $t=0$ Schwarz's theory predicts

$$
X_{1}(0)=-\left(\frac{\partial \ln s}{\partial \ln \bar{c}_{\mathrm{A}}}\right)_{P, c_{\mathrm{A}}{ }^{0}} \ll 1
$$

Accordingly, the following equation is also derived.

$$
X_{2}(0)=-\left(\frac{\partial \ln (s / q)}{\partial \ln \bar{c}_{\mathrm{A}}}\right)_{P, c_{\mathrm{A}} \mathrm{A}^{0}} \ll 1
$$

Thus for some initial part of the relaxation process the following relations may be accepted.

$$
\begin{aligned}
& X_{1}(t) \ll 1 \\
& X_{2}(t) \ll 1
\end{aligned}
$$

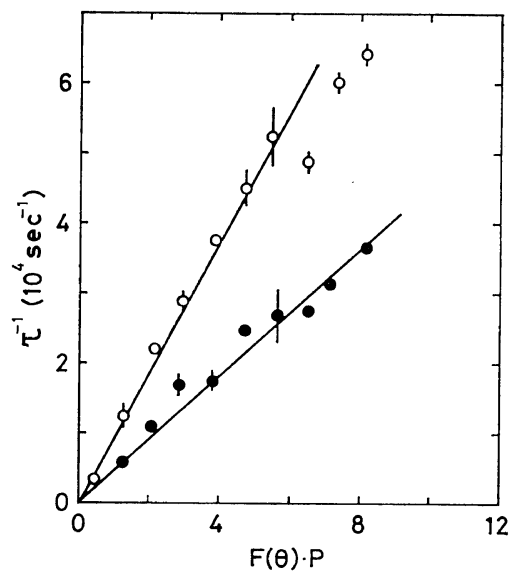

Figure 5. Plots of the reciprocal relaxation time, $(1 / \tau)$, vs. $F(\theta) P$ according to equation (18) at constant total weighing-in concentration of the dye, $c_{\mathrm{A}}{ }^{0}$ at $\mathrm{pH}$ 10: $\bigcirc, c_{\mathrm{A}}{ }^{0}=20 \mu M ; \bigcirc, c_{\mathrm{A}}{ }^{0}=50 \mu M$. The solid straight lines were drawn by the leastsquares procedure.

With the reciprocal mean relaxation time $\left(\tau^{-1}\right)$ the relaxation equation is described as follows

where

$$
\begin{aligned}
\tau^{-1} & =2 k_{\mathrm{R}} \bar{c}_{\mathrm{auu}}+f_{\mathrm{u}} k_{\mathrm{R}} \bar{c}_{\mathrm{uuu}} \\
& =k_{\mathrm{R}} g c_{\mathrm{A}}{ }^{0} F(\theta) P
\end{aligned}
$$

$$
F(\theta) \equiv 2 \sqrt{s / q} \sqrt{\theta(1-\theta)}-f_{\mathrm{u}}(1-\theta)
$$

Plots of $1 / \tau$ vs. $F(\theta) P$ at constant $c_{\mathrm{A}}{ }^{0}$ are shown in Figure 5, in which a fair degree of linearity indicates the model assumed above to be reasonable. The value of $f_{\mathrm{u}}$ was determined at about 0.7 so as to obtain the best straight line for the data. According to the Schwarz's theory this parameter is equal to no more than unity if the interaction does not involve any activation barrier. ${ }^{10}$ In this work, however, the value of $f_{\mathfrak{u}}$ was 0.7 , implying the existence of a low activation barrier. The rate constants, $k_{\mathrm{R}}$ and $k_{\mathrm{D}}$ obtained from the relation $K=k_{\mathrm{R}} / k_{\mathrm{D}}$ are summerized in Table II.

No remarkable dependence of the association rate constant $\left(k_{R}\right)$ on the degree of ionization can be seen. The value of dissociation rate constant $\left(k_{\mathrm{D}}\right)$ is almost constant between $\mathrm{pH}$ 6.5 and 8 but changes greatly at $\mathrm{pH} 10$. These facts are consistent with the dependences of the values of $g$ and $q$ on the $\mathrm{pH}$ and imply that the bound dyes should become more stable with 
Table II. Rate constants of the binding of toluidine blue to poly(acrylic acid) at $20^{\circ} \mathrm{C}$

\begin{tabular}{ccccc}
\hline pH & $\begin{array}{c}k_{\mathrm{R}}, \\
10^{8} M^{-1} \mathrm{sec}^{-1}\end{array}$ & $\begin{array}{c}k_{\mathrm{D}}, \\
10^{2} \mathrm{sec}^{-1}\end{array}$ & $\begin{array}{c}f_{\mathrm{u}} k_{\mathrm{R}}, \\
10^{8} M^{-1} \mathrm{sec}^{-1}\end{array}$ & $\begin{array}{c}f_{\mathrm{u}} q k_{\mathrm{D}}, \\
10^{3} \mathrm{sec}^{-1}\end{array}$ \\
\hline 6.5 & $1.3( \pm 0.2)$ & 2.7 & 0.91 & 3.8 \\
8 & $1.8( \pm 0.2)$ & 3.3 & 1.3 & 0.9 \\
10 & $1.4( \pm 0.2)$ & 0.82 & 0.98 & 4.7 \\
\hline
\end{tabular}

increasing the degree of ionization. From the fact that the value of dissociation rate constant $\left(f_{\mathrm{u}} q k_{\mathrm{D}}\right)$ for the nucleation process is almost constant with respect to changes in $\mathrm{pH}$, it is clear that the value of $k_{\mathrm{D}}$ is greatly affected by the degree of cooperativity.

The reaction model proposed here could be successfully applied to the experimental results. The contribution of the nucleation process to the chemical relaxation could not be neglected; the reason for this, however, is not entirely clear. It has been reported that the contribution of the nucleation process is negligible with a medium $P / D$ value and a sufficiently large $q$ value. $^{10}$ The values of $q$ determined spectrophotometrically in this work are not very small compared with those obtained in the case of $(\mathrm{Glu})_{n} \cdot{ }^{16}$ Nevertheless the contribution of the nucleation process appeared in the observed relaxation phenomena. This may be due to the fact that the condition of $\bar{c}_{\text {uau }} \ll \bar{c}_{\text {auu }}$ or $\bar{c}_{\text {aau }}$ is not fully recognized for some reason in the case of PAA. Thus the contribution of the nucleation process should be always taken into account irrespective of the magnitude of the value of $g$.

In the case of $(\mathrm{Glu})_{n}, k_{\mathrm{R}}$ was found to be about $10^{9} \mathrm{M}^{-1} \mathrm{sec}^{-1}$ which clearly indicated a diffusion-controlled reaction process, ${ }^{13,16}$ where as the value of $k_{\mathrm{R}}$ for PAA turned out to be about $10^{8} M^{-1} \mathrm{sec}^{-1}$ under the same experimental condition. The reason for this discrepancy is not apparent, but several possible explanations exist. First, it is expected that the rate constant for the diffusion-controlled reaction would take on a somewhat smaller value on account of the difference in the type of polymer. The rate constant for the diffusion-controlled process of the complex formation is given by

$$
k=4 \pi N r\left(D_{\mathrm{A}}+D_{\mathrm{B}}\right)\left(\frac{\theta}{\mathrm{e}^{\theta}-1}\right) 10^{-3} M^{-1} \sec ^{-1}
$$

and

$$
\theta=\frac{\left|z_{\mathrm{A}} z_{\mathrm{B}}\right| e_{0}^{2}}{\varepsilon \boldsymbol{k} T r}
$$

where $N$ is Avogadro's number, $D_{\mathrm{A}}$ and $D_{\mathrm{B}}$ are translational diffusion coefficient of the reactant $\mathrm{A}$ and $\mathrm{B}$, respectively, $z_{\mathrm{A}}$ and $z_{\mathrm{B}}$ are charge on the reactant $\mathrm{A}$ and $\mathrm{B}$, respectively, $e_{0}$ is electrostatic charge, $\varepsilon$ is dielectric constant, and $r$ is distance of closest approach of ions of the complex. ${ }^{23}$ In case of the interaction between the macromolecule and the small molecule, the term of the diffusion coefficient should be represented approximately only by that of the small molecule since the diffusion coefficient of the macromolecule is small compared with that of the small molecule. Using a dielectric constant of 80 , with the distance between ions in the complex being equal to $5-10 \AA$, and the diffusion coefficient value being equal to $0.5-1 \times 10^{-5}$ $\mathrm{cm}^{2} \mathrm{sec}^{-1}$, the value of $k$ is found to be $8.6 \times$ $10^{8}-2.6 \times 10^{9} \mathrm{M}^{-1} \mathrm{sec}^{-1}$. Thus the rate constant for the diffusion-controlled process of the complex formation between the macromolecule and the small molecule should be around $10^{9} \mathrm{M}^{-1} \mathrm{sec}^{-1}$ irrespective of the kind of macromolecule. Therefore, it can be supposed that the recombination process of toluidine blue to PAA is not diffusion-controlled, which is in apparent contrast to the $(\mathrm{Glu})_{n}$-toluidine-blue system.

Next, another reason for the cause of the difference in the value of $k_{\mathrm{R}}$ between these polymers is considered. In a polyelectrolyte having a small distance less than about $3 \AA$ between the adjacent side chains, such as PAA, Ikegami has reported that the second hydration region around a polyion is formed in addition to the intrinsic hydration with increase in the degree of ionization of carboxylic groups. ${ }^{24,25}$ In the case of a polyelectrolyte such as $(\mathrm{Glu})_{n}$, however, the second hydration region is not formed, since the distance between neighboring charged groups is more than $3 \AA^{14,25}$ If the toluidine blue binds to the second hydration region of PAA, the value of $k_{\mathrm{R}}$ would be the same as that for the diffusion-controlled process (there is no energy barrier). However, the fact that the value of $k_{\mathrm{R}}$ obtained in this work is 
Kinetic study of the PAA-Dye Complex Formation

smaller than that for the diffusion-controlled process is evidence of the existence of another reaction process. Therefore, if the toluidine blue binds to the surface of the intrinsic hydration region of PAA, the dye must destroy the second hydration region on binding to the PAA. For this reason, it is expected that the value of $k_{\mathrm{R}}$ will be smaller than that for the diffusion-controlled process. In addition, the expectation that the toluidine blue binds to the intrinsic hydration region of PAA is consistent with the suggestion that the dye binds to the polymer not directly but in the manner of an ionic atmosphere, obtained from the value of $g$ and its dependence on the degree of ionization.

In conclusion, the discrepancy of the value of the rate constant for the recombination process between PAA and $(\mathrm{Glu})_{n}$ can be attributed to the difference in the structure of the hydration region of the polymer.

\section{REFERENCES}

1. A. R. Peacocke and J. N. H. Skerrett, Trans. Faraday Soc., 52, 261 (1956).

2. D. F. Bradley and M. K. Wolf, Proc. Natl. Acad. Sci. U.S.A., 45, 944 (1959).

3. L. Stryer and E. R. Blout, J. Am. Chem. Soc., 83, 1411 (1961).

4. R.W. Armstrong, T. Kurucsev, and U.P. Strauss, ibid., 92, 3174 (1970).

5. M. Dourlent and C. Hélène, Eur. J. Biochem., 23, 86 (1971).
6. G. G. Hammes and C. D. Hubbard, J. Phys. Chem., 70, 1615 (1966).

7. G. G. Hammes and C. D. Hubbard, ibid., 70, 2886 (1966).

8. H. J. Li and D. M. Crothers, J. Mol. Biol., 39, 461 (1969).

9. R. Bittman, ibid., 46, 251 (1969).

10. G. Schwarz, Eur. J. Biochem., 12, 442 (1970).

11. M. Dourlent, Biopolymers, 14, 1717 (1975).

12. G. Schwarz, S. Klose, and W. Balthasar, Eur. J. Biochem., 12, 454 (1970).

13. G. Schwarz and W. Balthasar, ibid., 12, 461 (1970).

14. G. Schwarz and S. Klose, ibid., 29, 249 (1972).

15. V. Vitagliano, J. Phys. Chem., 77, 1922 (1973).

16. H. Ushio, T. Yasunaga, T. Sano, and Y. Tsuji, Biopolymers, 15, 187 (1976).

17. A. Takahashi, N. Hayashi, and I. Kagawa, Kogyo Kagaku Zassi (J. Chem. Soc. Japan, Ind. Chem. Sect.) 60, 1059 (1957).

18. Y. Tsuji, T. Yasunaga, T. Sano, and H. Ushio, J. Am. Chem. Soc., 98, 813 (1976).

19. L. Popa and R. Repanovici, Biochim. Biophys. Acta, 182, 158 (1969).

20. M. Dourlent, J. Hogrel, and C. Hélène, $J$. Am. Chem. Soc., 96, 3398 (1974).

21. H. Kawabe and M. Yanagita, Bull. Chem. Soc. Japan, 42, 3109 (1969).

22. G. Ilgenfritz, Ph. D. Dissertation, GeorgAugust Univ., Göttingen, (1966).

23. G. Schwarz and A. Seelig-Löffler, Biochim. Biophys. Acta, 379, 125 (1975).

24. A. Ikegami, J. Polym. Sci., Part A, 2, 907 (1964).

25. A. Ikegami, Biopolymers, 6, 431 (1968). 